\title{
Penta-quark baryon in anisotropic lattice QCD
}

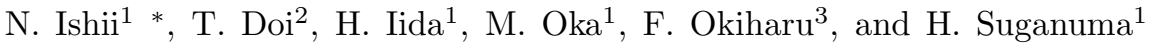 \\ 1 Department of Physics, H-27, Faculty of Science, \\ Tokyo Institute of Technology, Meguro, Tokyo 152-8551, Japan \\ ${ }^{2}$ RIKEN BNL Research Center, Brookhaven National Laboratory, Upton, New York 11973, USA and \\ ${ }^{3}$ Department of Physics, Faculty of Science and Technology, \\ Nihon University, 1-8-14 Kanda-Surugadai, Chiyoda, Tokyo 101-8308, Japan
}

\begin{abstract}
The penta-quark $(5 \mathrm{Q})$ baryon is studied in anisotropic quenched lattice $\mathrm{QCD}$ with renormalized anisotropy $a_{s} / a_{t}=4$ for a high-precision mass measurement. The standard Wilson action at $\beta=5.75$ and the $O(a)$ improved Wilson quark action with $\kappa=0.1210(0.0010) 0.1240$ are employed on a $12^{3} \times 96$ lattice. Contribution of excited states is suppressed by using a smeared source. We investigate both the positive- and negative-parity $5 \mathrm{Q}$ baryons with $I=0$ and spin $J=1 / 2$ using a non-NK-type interpolating field. After chiral extrapolation, the lowest positive-parity state is found to have a mass, $m_{\Theta}=2.25 \mathrm{GeV}$, which is much heavier than the experimentally observed $\Theta^{+}(1540)$. The lowest negative-parity $5 \mathrm{Q}$ appears at $m_{\Theta}=1.75 \mathrm{GeV}$, which is near the s-wave NK threshold. To distinguish spatially-localized $5 \mathrm{Q}$ resonances from NK scattering states, we propose a new general method imposing a "Hybrid Boundary Condition (HBC)", where the NK threshold is artificially raised without affecting compact five-quark states. The study using the HBC method shows that the negative-parity state observed on the lattice is not a compact 5Q but an s-wave NK-scattering state.

PACS numbers: 12.38.Gc, 12.39.Mk, 14.20.-c, 14.20.Jn
\end{abstract}

\section{INTRODUCTION}

Quantum chromodynamics (QCD) is the fundamental theory of hadron physics. It specifies elementary interactions at the level of quarks and gluons, which serve as building-blocks to construct complicated interactions among hadrons. At high energy, the QCD coupling constant diminishes due to asymptotic freedom, and perturbative QCD works well. At low energy, however, with the growth of the QCD coupling constant, perturbation theory breaks down, and the system is dominated by nonperturbative effects.

One of the most important features of nonperturbative QCD is color confinement, i.e., all hadrons are formed as color singlet combinations of quarks and gluons. In the conventional picture, mesons consist of a quark and an antiquark, while baryons are made of three quarks. QCD, however, allows more general hadrons, often called exotic hadrons, which contain extra pairs of quark and antiquark, or gluons. Possible candidates include multiquark hadrons, glueballs, and hybrid hadrons. Experimental searches found no manifestly exotic hadrons before the year 2002. Therefore, the discovery of the manifestly exotic narrow resonance $\Theta^{+}(1540)$ by LEPS group at SPring-8 was an epoch making event in the hadron physics [1].

$\Theta^{+}$is confirmed to have baryon number $B=1$, charge $Q=+1$ and strangeness $S=+1$. It has to contain at least one anti-strange quark and its simplest configuration is $u u d d \bar{s}$. Therefore, $\Theta^{+}$is a manifestly exotic

\footnotetext{
*E-mail : ishii@rarfaxp.riken.jp
}

penta-quark state.

The experimental search [1] of $\Theta^{+}$was motivated by a theoretical prediction given in Ref. 2]. It is noted that $\Theta^{+}$had been considered several times in the history 3 , 4, 5]. The discovered peak in the $n K^{+}$invariant mass is centered at $1.54 \pm 0.01 \mathrm{GeV}$ with a width smaller than 25 MeV. A number of groups [6, 7, 8, 9] confirmed the LEPS discovery, among which Ref. 8] claims that $\Theta^{+}$must be isoscalar, since no $\Theta^{++}$is observed in the $p K^{+}$invariant mass spectrum.

Numerous theoretical studies of penta-quark baryons have appeared since its discovery $5,10,11,12,13,14$, 15, 16, 17, 18, 19, 20, 21, 22, 23, 24, 25, 26, 27, 28, 29, 30, $31,32,33,34,35,36$. One of the important issues is the spin and parity of $\Theta^{+}$. Although $J^{\pi}=\frac{1}{2}^{+}$is suggested in the original work of Ref.[2] based on the chiral soliton model, there is no direct experimental information available on spin and parity 37]. An educated guess for the spin is $J=1 / 2$, since the color-magnetic interaction favors smaller spin states in general. There is, however, no consensus on parity at all. Experimental determination of the parity of $\Theta^{+}$happens to be quite challenging [38], while opinions are divided in theory [39, 40].

Positive parity is supported by various model calculations, i.e., soliton models [2, 5, 10, 11, 12, 41], chiral bag model 13], the Jaffe-Wilczek's diquark model 14], the Karliner-Lipkin's diquark-triquark model [15], some quark model calculations 16, 17, 18, 19, 20], and other model calculations [21, 22]. Negative parity is supported by some other quark model calculations [3, 4, 23, 24, 25], and QCD sum rules [28].

There are several lattice QCD studies of penta-quarks available [42, 43, 44, 45, 46, 47], but they have not reached a consensus yet. Except for Ref. 44], all these cal- 
culations support that negative parity states are lighter than the positive parity ones and that positive-parity penta-quarks may appear at the mass above $2 \mathrm{GeV}$. Refs. 42, 43 claimed existence of a bound negative-parity penta quark, whereas Ref. 45] has observed no evidence for narrow resonances. Naively, the negative result by Ref. 45] may be understood as a natural consequence of their choice of the NK-type interpolating field, since it is expected to couple strongly to the NK continuum state. Therefore, under these circumstances, it is important to use various interpolating fields and accumulate more data to give a precise prediction.

The aim of this paper is twofold. (i) We provide a high-precision data on the penta-quark baryon $\Theta^{+}$by using the anisotropic lattice QCD. (ii) We propose a new method, which enables us to distinguish compact pentaquarks from NK scattering states and apply it to the negative-parity $\Theta^{+}$.

The paper is organized as follows. In Sect. III we discuss our choice of the interpolating field for the pentaquark $\Theta^{+}$, and introduce the parity projection. We adopt the standard Wilson gauge action at $\beta=5.75$ on the $12^{3} \times 96$ lattice with the renormalized anisotropy $\xi \equiv a_{s} / a_{t}=4$. The use of the anisotropic lattice is known to serve as a powerful tool for high-precision measurements of temporal correlators [48, 49, 50, 51]. For quark action, we adopt $O(a)$-improved Wilson (clover) action with four values of the hopping parameter as $\kappa=0.1210(0.0010) 0.1240$. We also employ a smeared operator to enhance the low-lying spectra.

In Sect. III we present our numerical results for both positive and negative parity channels. For both cases, we observe a rather stable plateau in the effective mass plot. For the positive parity case, we obtain, after the chiral extrapolation, $m_{\Theta}=2.25 \mathrm{GeV}$, which is much heavier than the observed $\Theta^{+}(1540)$. For the negative parity case, we obtain $m_{\Theta}=1.75 \mathrm{GeV}$, which is rather close to the empirical value. Our data thus suggest that the negative-parity state is the ground state of the pentaquark spectrum.

To clarify whether this negative parity state is really a localized resonance or not, we propose a new general method with a new "hybrid boundary condition (HBC)" for the quark fields. Sect. IV] is devoted to description of the $\mathrm{HBC}$ method. In the $\mathrm{HBC}$ method, the spatial boundary condition on the quark fields are artificially taken so that $\mathrm{N}$ and $\mathrm{K}$ must have non-vanishing spatial momenta, while it does not affect localized penta-quark states. As a consequence, the HBC raises the s-wave NK threshold by a few hundred $\mathrm{MeV}$ without changing the mass of a compact resonance state, $\Theta^{+}$.

By applying the $\mathrm{HBC}$ method, we investigate the negative-parity state further. Particularly, we examine difference of the spectra between the two boundary conditions. We find that, in the effective mass plots, the plateau is raised by about the same amount as the shift of the NK-thresholds. We therefore conclude that there is no localized resonance state below
$\sqrt{m_{\mathrm{N}}^{2}+\vec{p}_{\min }^{2}}+\sqrt{m_{\mathrm{K}}^{2}+\vec{p}_{\min }^{2}}$ with $\left|\vec{p}_{\min }\right|=\sqrt{3} \pi / L$. Thus the state observed under the standard boundary condition turns out to be an NK scattering state.

In Sect. $\nabla$ a summary is given and possible implications of our results are presented.

\section{INTERPOLATING FIELD AND PARITY PROJECTION}

We consider a non-NK type interpolating field for the penta-quark $\Theta^{+}$as

$$
O_{\alpha} \equiv \epsilon_{a b c} \epsilon_{a d e} \epsilon_{b f g}\left(u_{d}^{T} C \gamma_{5} d_{e}\right)\left(u_{f}^{T} C d_{g}\right)\left(C \bar{s}_{c}^{T}\right)_{\alpha}
$$

where $\alpha$ denotes the Dirac index, and $a-g$ are color indices. $C \equiv \gamma_{4} \gamma_{2}$ denotes charge conjugation matrix. The quantum number of Eq. (11) is spin $J=1 / 2$ and isospin $I=0$. It has an advantage that it cannot be decomposed into a product of $\mathrm{N}$ and $\mathrm{K}$ in the non-relativistic limit. Hence, we may expect that its coupling to NK scattering states is rather weak. This is the crucial difference between our calculation and that of Ref. [45], who adopted the NK type interpolating field, which is expected to couple to NK scattering states strongly. The NK type interpolating field would not be an appropriate choice in studying the narrow penta-quark resonance in this sense.

We present Fierz rearrangements of Eq. (1) in Appendix. A] We can explicitly see that the NK component in the correlation function is suppressed by a factor 1/16 compared to that from the NK-type operator. This non-NK type interpolating field Eq. (1) was adopted in Refs. 28, 43, 44].

Under the spatial reflection, the quark fields transform as $q(t, \vec{x}) \rightarrow \gamma_{4} q(t,-\vec{x})$. Therefore, Eq. (1) transforms as

$$
O(t, \vec{x}) \rightarrow+\gamma_{4} O(t,-\vec{x}) .
$$

This means that the intrinsic parity of Eq. (1) is positive. Although the intrinsic parity of Eq. (1) is positive, it couples to negative parity states as well [52]. In the Dirac representation, one sees that the upper (lower) component creates positive (negative) parity states. In addition, in lattice formulation with limited temporal extension, it may create backwardly-propagating state with opposite parity. Since the parity projection of baryon is thus involved, we explain our strategy of parity projection.

We consider the asymptotic behavior of the zeromomentum projected correlator as

$$
G_{\alpha \beta}(t) \equiv \frac{1}{V} \sum_{\vec{x}}\left\langle O_{\alpha}(t, \vec{x}) \bar{O}_{\beta}(0, \overrightarrow{0})\right\rangle
$$

in large $t$ and large $N_{t}-t$ region. ( $V$ and $N_{t}$ refer to the spatial volume and the temporal size of the lattice, respectively.) In this region, the correlator is assumed to 
be dominated by the lowest-lying states of each parity, and is decomposed into two parts in the following manner 52]:

$$
\begin{aligned}
G^{( \pm)}(t) & \equiv P_{+}\left(C_{+} e^{-m_{+} t} \pm C_{-} e^{-m_{-}\left(N_{t}-t\right)}\right) \\
& +P_{-}\left(C_{-} e^{-m_{-} t} \pm C_{+} e^{-m_{+}\left(N_{t}-t\right)}\right)
\end{aligned}
$$

where the choice of " \pm " depends on the boundary condition of quark fields in imaginary time, i.e., "+" for anti-periodic boundary condition and "-" for periodic boundary condition. $m_{+}$and $m_{-}$refer to the energies of lowest-lying states in positive and negative parity channels, respectively. $P_{ \pm} \equiv\left(1 \pm \gamma_{4}\right) / 2$ work as projection matrices onto the "upper" and the "lower" Dirac subspaces, respectively.

We consider the first term in Eq. (4). In the region $0 \ll t \ll N_{t} / 2, C_{+} e^{-m_{+} t}$ is expected to dominate the correlator, whereas, in the region $N_{t} / 2 \ll t \ll N_{t}$, $C_{-} e^{-m_{-}\left(N_{t}-t\right)}$ is expected to dominate the correlator. In the intermediate region, i.e., $t \sim N_{t} / 2$, the contributions from the both terms cannot be negligible. The second term in Eq. (4) behaves in a similar manner. In this way, by confining ourselves to the region $0 \ll t \ll N_{t} / 2$, and by applying the projection matrices $P^{( \pm)}$to $G(t)$, we can pick up the positive and negative parity states separately.

One may point out that the exact parity projection can be achieved by summing up $G^{(+)}$and $G^{(-)}$, because the backwardly-propagating contributions cancel. This is often used to project parity for ordinary "three-quark" baryons at quenched level. However, in the case of pentaquark, this may not work even in quenched level, because the five-quark system can decompose into $\mathrm{N}$ and $\mathrm{K}$ so that $\mathrm{N}$ may propagate forward whereas $\mathrm{K}$ may propagate backward or vice versa [4].

\section{NUMERICAL RESULT ON $\Theta^{+}$MASS}

\section{A. Lattice parameter set}

To generate gauge field configurations, we use the standard plaquette action on the anisotropic lattice of the size $12^{3} \times 96$ as

$$
\begin{aligned}
S_{\mathrm{G}} & =\frac{\beta}{N_{c}} \frac{1}{\gamma_{\mathrm{G}}} \sum_{s, i<j \leq 3} \operatorname{Re} \operatorname{Tr}\left\{1-P_{i j}(s)\right\} \\
& +\frac{\beta}{N_{c}} \gamma_{\mathrm{G}} \sum_{s, i \leq 3} \operatorname{Re} \operatorname{Tr}\left\{1-P_{i 4}(s)\right\}
\end{aligned}
$$

where $P_{\mu \nu}(s) \in \mathrm{SU}(3)$ denotes the plaquette operator in the $\mu$ - $\nu$-plane. The lattice parameter and the bare anisotropy parameter are fixed as $\beta \equiv 2 N_{c} / g^{2}=5.75$ and $\gamma_{\mathrm{G}}=3.2552$, respectively, which reproduce the renormalized anisotropy as $\xi \equiv a_{s} / a_{t}=4$ [48]. Adopting the pseudo-heat-bath algorithm, we pick up gauge field configurations every 500 sweeps after skipping 10,000 sweeps for the thermalization. We use totally 504 gauge field configurations to construct the temporal correlators. The lattice spacing is determined from the static quark potential adopting the Sommer parameter $r_{0}^{-1}=395$ $\mathrm{MeV}\left(r_{0} \sim 0.5 \mathrm{fm}\right)$ as $a_{s}^{-1}=1.100(6) \mathrm{GeV}\left(a_{s} \simeq\right.$ $0.18 \mathrm{fm})$. Hence the lattice size $12^{3} \times 96$ amounts to $(2.15 \mathrm{fm})^{3} \times(4.30 \mathrm{fm})$ in the physical unit.

For quark fields $\psi$ and $\bar{\psi}$, we adopt the $O(a)$-improved Wilson (clover) action on the anisotropic lattice as 49]

$$
\begin{aligned}
& S_{\mathrm{F}} \equiv \sum_{x, y} \bar{\psi}(x) K(x, y) \psi(y), \\
& K(x, y) \equiv \delta_{x, y}-\kappa_{t}\left\{\begin{array}{l}
\left(1-\gamma_{4}\right) U_{4}(x) \delta_{x+\hat{4}, y} \\
+ \\
\left.\quad+\left(1+\gamma_{4}\right) U_{4}^{\dagger}(x-\hat{4}) \delta_{x-\hat{4}, y}\right\}
\end{array}\right. \\
&-\kappa_{s} \sum_{i}\left\{\begin{array}{l}
\left(r-\gamma_{i}\right) U_{i}(x) \delta_{x+\hat{i}, y} \\
\left.\quad+\left(r+\gamma_{i}\right) U_{i}^{\dagger}(x-\hat{i}) \delta_{x-\hat{i}, y}\right\}
\end{array}\right. \\
&-\kappa_{s} c_{E} \sum_{i} \sigma_{i 4} F_{i 4} \delta_{x, y}-r \kappa_{s} c_{B} \sum_{i>j} \sigma_{i j} F_{i j} \delta_{x, y},
\end{aligned}
$$

where $\kappa_{s}$ and $\kappa_{t}$ denote the spatial and temporal hopping parameters, respectively. $F_{\mu \nu}$ denotes the field strength, which is defined through the standard cloverleaf-type construction. $r$ denotes the Wilson parameter. $c_{E}$ and $c_{B}$ denote the clover coefficients. To achieve the tadpole improvement, the link variables are rescaled as $U_{i}(x) \rightarrow U_{i}(x) / u_{s}$ and $U_{4}(x) \rightarrow U_{4}(x) / u_{t}$, where $u_{s}$ and $u_{t}$ denote the mean-field values of the spatial and temporal link variables, respectively [49, 50]. This is equivalent to the redefinition of the hopping parameters as the tadpole-improved ones (with tilde), i.e., $\kappa_{s}=\tilde{\kappa}_{s} / u_{s}$ and $\kappa_{t}=\tilde{\kappa}_{t} / u_{t}$. The anisotropy parameter is defined as $\gamma_{F} \equiv \tilde{\kappa}_{t} / \tilde{\kappa}_{s}$, which coincides with the renormalized anisotropy $\xi=a_{\sigma} / a_{\tau}$ for sufficiently small quark mass at the tadpole-improved level [49]. For given $\kappa_{s}$, the four parameters $r, c_{E}, c_{B}$ and $\kappa_{s} / \kappa_{t}$ should be, in principle, tuned so that "Lorentz symmetry" holds up to discretization errors of $O\left(a^{2}\right)$. Here, $r, c_{E}$ and $c_{B}$ are fixed by adopting the tadpole improved tree-level values as

$$
r=\frac{1}{\xi}, \quad c_{E}=\frac{1}{u_{\sigma} u_{\tau}^{2}}, \quad c_{B}=\frac{1}{u_{\sigma}^{3}} .
$$

Only the value of $\kappa_{\sigma} / \kappa_{\tau}\left(=\gamma_{F} \cdot\left(u_{\sigma} / u_{\tau}\right)\right)$ is tuned nonperturbatively by using the meson dispersion relation [49]. It is convenient to define $\kappa$ as

$$
\frac{1}{\kappa} \equiv \frac{1}{\tilde{\kappa}_{\sigma}}-2\left(\gamma_{F}-3 r-4\right),
$$

then the bare quark mass is expressed as $m_{0}=\frac{1}{2}(1 / \kappa-8)$ in the spatial lattice unit in the continuum limit. This $\kappa$ plays the role of the hopping parameter " $\kappa$ " in the isotropic formulation. For detail, see Refs. [49, 50], from which we take the lattice parameters. The values of the lattice parameters are summarized in Table凹 


\begin{tabular}{ccccccccccc}
\hline \hline$\beta$ & $\gamma_{\mathrm{G}}$ & $a_{s} / a_{t}$ & $a_{s}^{-1}[\mathrm{GeV}]$ & Size & $N_{\text {conf }}$ & $u_{\sigma}$ & $u_{\tau}$ & $\gamma_{F}$ & $\kappa_{c}$ & Values of $\kappa$ \\
\hline 5.75 & 3.2552 & 4 & $1.100(6)$ & $12^{3} \times 96$ & 504 & $0.7620(2)$ & $0.9871(0)$ & 3.909 & $0.12640(5)$ & $0.1240,0.1230,0.1220,0.1210$ \\
\hline \hline
\end{tabular}

TABLE I: Parameters of the lattice simulation. The spatial lattice spacing $a_{\sigma}$ is determined with $r_{0}^{-1}=385 \mathrm{MeV}$ for the Sommer parameter. The mean-field values of link variables $\left(u_{\sigma}\right.$ and $\left.u_{\tau}\right)$ are defined in the Landau gauge. $\kappa_{c}$ denotes the critical value of $\kappa$.

\begin{tabular}{llllll}
\hline \hline$\kappa$ & 0.1210 & 0.1220 & 0.1230 & 0.1240 & $\kappa_{\text {phys. }}$ \\
\hline$m_{\pi}$ & $1.005(2)$ & $0.898(2)$ & $0.784(2)$ & $0.656(3)$ & 0.140 \\
$m_{\rho}$ & $1.240(3)$ & $1.161(3)$ & $1.085(4)$ & $1.011(5)$ & $0.850(7)$ \\
$m_{K}$ & $0.845(2)$ & $0.785(2)$ & $0.723(2)$ & $0.656(3)$ & $0.530(4)$ \\
$m_{N}$ & $1.878(5)$ & $1.744(5)$ & $1.604(5)$ & $1.460(6)$ & $1.173(9)$ \\
\hline \hline
\end{tabular}

TABLE II: Masses of $\pi, \rho, \mathrm{K}$ and $\mathrm{N}$ for each hopping parameter $\kappa$ in the physical unit GeV. $\kappa_{\text {phys. }} \simeq 0.1261$ denotes the value of $\kappa$ which achieves $m_{\pi} \simeq 0.14 \mathrm{GeV}$.

We adopt four values of the hopping parameter as $\kappa=0.1210,0.1220,0.1230$ and 0.1240 , which correspond to $m_{\pi} / m_{\rho}=0.81,0.77,0.72$ and 0.65 , respectively. For temporal direction, we impose anti-periodic boundary condition on all the quark fields. For spatial directions, unless otherwise indicated, we impose periodic boundary condition on all the quarks. We refer to this boundary condition as "standard (spatial) $B C$ ".

By keeping $\kappa_{s}=0.1240$ fixed for s quark, and by changing $\kappa=0.1210-0.1240$ for $\mathrm{u}$ and $\mathrm{d}$ quarks, we perform the chiral extrapolation to the physical quark mass region. In the following part of the paper, we will use

$$
\left(\kappa_{s}, \kappa\right)=(0.1240,0.1220),
$$

as a typical set of hopping parameters in presenting correlators and effective mass plots. For convenience, we summarize masses of $\pi, \rho, \mathrm{K}$ and $\mathrm{N}$ for each hopping parameter $\kappa$ together with their chirally extrapolated values in Table Here, the chiral extrapolations of these particles are performed with a linear function in $m_{\pi}^{2}$. Unless otherwise indicated, we adopt jackknife prescription to estimate statistical errors.

In order to enhance the low-energy contributions, we use a smeared source. This is achieved by employing a spatially extended interpolating field of the gaussian size $\rho \simeq 0.4 \mathrm{fm}$ in the Coulomb gauge, which is obtained by replacing the quark fields $q(x)$ in Eq. (10) by the smeared quark fields $q_{\text {smear }}(x)$ defined as

$$
q_{\text {smear }}(t, \vec{x}) \equiv \mathcal{N} \sum_{\vec{y}} \exp \left\{-\frac{|\vec{x}-\vec{y}|^{2}}{2 \rho^{2}}\right\} q(t, \vec{y}),
$$

where $\mathcal{N}$ is an appropriate normalization factor. For practical use, we extend Eq. (10) appropriately so as to fit a particular choice of the spatial boundary condition. In this paper, we present correlators with a smeared source and a point sink.

\begin{tabular}{llllll}
\hline \hline$\kappa$ & 0.1210 & 0.1220 & 0.1230 & 0.1240 & empirical \\
\hline s-wave & 2.723 & 2.528 & 2.327 & 2.116 & 1.440 \\
p-wave & 2.987 & 2.809 & 2.629 & 2.442 & 1.865 \\
HBC & 2.924 & 2.743 & 2.558 & 2.367 & 1.770 \\
\hline \hline
\end{tabular}

TABLE III: Numerical values of NK thresholds (Eq. [13) for each hopping parameter $\kappa$ in the physical unit $\mathrm{GeV}$, when spatial lattice of the size is $L \simeq 2.15 \mathrm{fm}$. The rightmost column indicated by "empirical" corresponds to the thresholds calculated for the physical masses of $\mathrm{N}$ and $\mathrm{K}, m_{N}=0.94$ $\mathrm{GeV}$ and $m_{K}=0.5 \mathrm{GeV}$. The first and the second rows show the NK thresholds for the s-wave and p-wave states, respectively, when the standard $\mathrm{BC}$ is imposed. The third row shows the NK thresholds for the HBC. In the HBC, the s-wave and p-wave NK thresholds coincide.

\section{B. NK scattering states for standard BC}

The NK scattering state with zero total momentum is obtained as $\mathrm{J}=1 / 2$-projection of the following state:

$$
|N(\vec{p}, s) K(-\vec{p})\rangle \pm|N(-\vec{p}, s) K(\vec{p})\rangle,
$$

where "+" is for s-wave, and "-" for p-wave. $s$ denotes the spin degrees of freedom of the nucleon. In finite spatial box of the size $L^{3}$, the allowed momentum is quantized as

$$
\vec{p}=\frac{2 \pi}{L} \vec{n}, \quad \vec{n} \in \mathbb{Z}^{3},
$$

due to the periodic boundary condition. By neglecting the interaction between $\mathrm{N}$ and $\mathrm{K}$, the energy of this state is approximated as

$$
E(\vec{p}) \simeq \sqrt{m_{K}^{2}+\vec{p}^{2}}+\sqrt{m_{N}^{2}+\vec{p}^{2}} .
$$

For s-wave, the NK threshold is expressed as $E_{\text {th }} \equiv$ $m_{K}+m_{N}$. On the other hand, for p-wave, since Eq. (11) vanishes for $\vec{p}=\overrightarrow{0}$, the NK threshold starts from $E_{\mathrm{th}} \equiv$ $E\left(\vec{p}_{\text {min }}\right)$ associated with the minimum momentum

$$
\left|\vec{p}_{\min }\right|=\frac{2 \pi}{L} .
$$

Numerical values of these NK thresholds for each hopping parameter $\kappa$ are presented in Table $\amalg$.

\section{The correlators for $\Theta^{+}$}

In Fig. 11 we show the effective mass plot for the positive parity channel, which is obtained from the correlator 


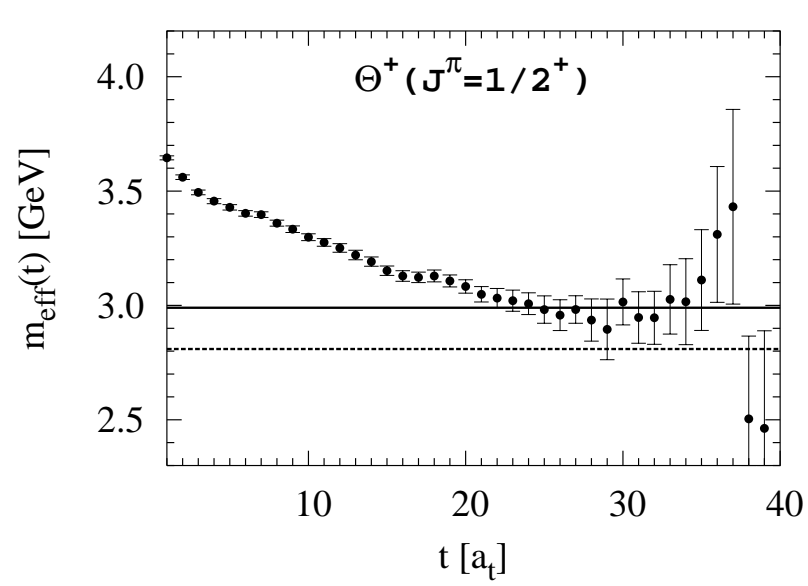

FIG. 1: The effective mass plot of positive parity $\Theta^{+}$. Eq. (9) is adopted as a typical set of hopping parameters. The statistical error is obtained with jackknife error estimate. The solid line denotes the result of the single-exponential fit performed over the interval $25 \leq t \leq 35$. The dotted line denotes the p-wave NK threshold energy corresponding to the spatial lattice size $L \simeq 2.15 \mathrm{fm}$.

with a smeared source and a point sink adopting Eq. (9). The dotted line denotes the p-wave NK threshold for the spatial lattice size $L \simeq 2.15 \mathrm{fm}$.

The effective mass is defined as

$$
m_{\mathrm{eff}}(t) \equiv \log \left(\frac{G(t)}{G(t+1)}\right)
$$

where $G(t)$ denotes the correlator. At sufficiently large $t$, contributions from excited states diminish, and the correlator is dominated by a single state with energy $m$ as $G(t) \sim A e^{-m t}$. Then Eq. (15) gives a constant effective mass as $m_{\text {eff }}(t) \sim m$. Thus a plateau can be served as an indicator of the single-state saturation. One is then allowed to perform the single-exponential fit in the plateau region.

In the region $0 \leq t \lesssim 25$ in Fig. 1 the effective mass $m_{\text {eff }}(t)$ decreases monotonically, which implies that the higher spectral contributions are gradually reduced. We find a plateau in the interval $25 \lesssim t \lesssim 35$, where the single-state dominance is expected to be achieved. Beyond $t \sim 35$, the data becomes too noisy. In addition, it is expected to receive the contribution from backwardlypropagating negative parity state as mentioned before. Hence, we simply neglect the data for $t \gtrsim 35$, and perform the single-exponential fit of the correlator with $f(t)=A e^{-m t}$ in the plateau region, $25 \leq t \leq 35$. The solid line denotes the best-fit result.

In Fig. 2 we show the effective mass plot for the negative parity channel adopting Eq. (9). The dotted line denotes s-wave NK threshold. In the small $t$ region, i.e., $t \lesssim 25, m_{\text {eff }}(t)$ decreases monotonically, and we see a rather stable plateau at $25 \lesssim t \lesssim 35$. The single exponential fit at $25 \leq t \leq 35$ gives the solid line.

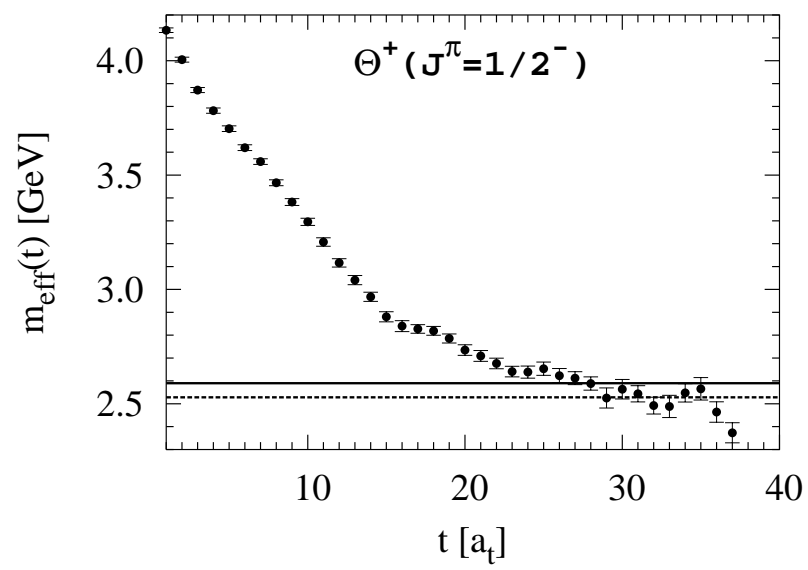

FIG. 2: The effective mass plot for negative parity $\Theta^{+}$for the typical set of hopping parameters (Eq. (9)). The solid line denotes the result of the single-exponential fit performed over the interval $25 \leq t \leq 35$. The dotted line denotes the s-wave NK threshold.

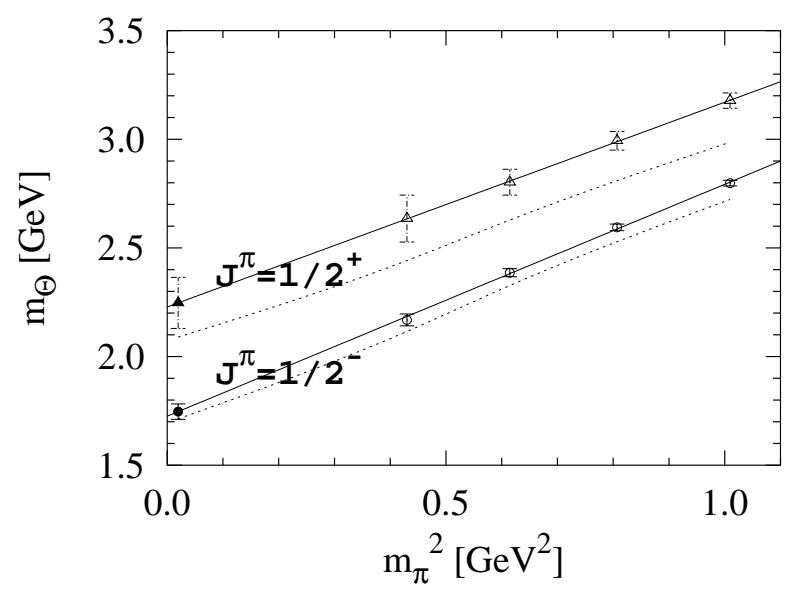

FIG. 3: $m_{\Theta}$ for both parity states plotted against $m_{\pi}^{2}$. The triangles denote positive parity, while the circles denote negative parity. The open symbols denote the direct lattice QCD data, whereas the closed symbols denote the results of the chiral extrapolation. The dotted lines indicate the NK thresholds for p-wave(upper) and s-wave(lower) cases.

\begin{tabular}{llllll}
\hline \hline$\kappa$ & 0.1210 & 0.1220 & 0.1230 & 0.1240 & $\kappa_{\text {phys. }}$ \\
\hline + parity & $3.18(4)$ & $2.99(4)$ & $2.80(6)$ & $2.64(11)$ & $2.25(12)$ \\
- parity & $2.80(1)$ & $2.59(2)$ & $2.39(2)$ & $2.17(3)$ & $1.75(4)$ \\
\hline \hline
\end{tabular}

TABLE IV: The masses of $\Theta^{+}$for each value of $\kappa$. The first line corresponds to the positive parity state, and the second line to the negative parity state. $\kappa_{\text {phys. }} \simeq 0.1261$ denotes the value of $\kappa$ which achieve $m_{\pi} \simeq 0.14 \mathrm{GeV}$. 
As mentioned before, $\kappa=0.1240$ is kept fixed for $\mathrm{s}$ quark, and $\kappa=0.1210-0.1240$ are varied for $\mathrm{u}$ and $\mathrm{d}$ quarks to perform the chiral extrapolation. In Fig. 3] the masses of positive (triangle) and negative (circle) parity $\Theta^{+}$are plotted against $m_{\pi}^{2}$. The open symbols denote the direct lattice data. Since these data behave almost linearly in $m_{\pi}^{2}$, the chiral extrapolation is performed with a linear function. Such a linear behavior against $m_{\pi}^{2}$ is also observed for ordinary non-PS mesons and baryons [50]. The closed symbols denote the results of chiral extrapolation. For convenience, we show p-wave (upper) and s-wave (lower) NK threshold with dotted lines.

For the positive parity state, the chiral extrapolation leads to $m_{\Theta}=2.25 \mathrm{GeV}$, which is much heavier than the experimentally observed $\Theta^{+}(1540)$. For negative parity state, on the other hand, the chiral extrapolation leads to $m_{\Theta}=1.75 \mathrm{GeV}$, which is rather close to the empirical value. Our lattice QCD results thus support that the negative parity state is the lowest of the $\Theta^{+}$spectrum. Table IV summarizes masses of $\Theta^{+}$of both parities for each hopping parameter together with their chirally extrapolated values.

\section{THE NEW METHOD TO DISTINGUISH $\Theta^{+}$ AND NK}

\section{A. The hybrid boundary condition (HBC)}

In the p-wave NK scattering states, $\mathrm{N}$ and $\mathrm{K}$ have nonzero minimum momenta as $\left|\vec{p}_{\min }\right|=2 \pi / L$ due to the finiteness of the spatial box. As a consequence, the NK threshold is raised by a few hundred $\mathrm{MeV}$ depending on the spatial volume. In studies of positive parity $\Theta^{+}$, we can utilize this volume dependence to distinguish localized resonances from NK-scattering states. In contrast, in studies of negative parity $\Theta^{+}$, NK scattering states are of s-wave, and $\mathrm{N}$ and $\mathrm{K}$ can have zero momenta, i.e., $\left|\vec{p}_{\text {min }}\right|=0$. Therefore, the NK threshold has no volume dependence, i.e., $m_{\mathrm{N}}+m_{\mathrm{K}}$, which is less convenient to distinguish $\Theta^{+}$from NK scattering states.

It would be of great worth, if we could find some prescription to raise the s-wave NK threshold by changing the spatial volume. This can be achieved by twisting the spatial boundary condition in a flavor dependent manner as follows. We impose the anti-periodic boundary condition on $\mathrm{u}$ and $\mathrm{d}$ quarks, whereas the periodic boundary condition on s quark. (See Table $\mathbf{V}$ ) We will refer to this spatial boundary condition as "hybrid boundary condition $(H B C)$ ".

As a consequence of the HBC, hadrons feel the boundary condition in their own ways. Since N(uud, udd) and $\mathrm{K}(u \bar{s}, d \bar{s})$ contain odd numbers of the $\mathrm{u}$ and $\mathrm{d}$ quarks, they are subject to the anti-periodic boundary condition. In contrast, since $\Theta^{+}(u u d d \bar{s})$ contain even numbers of $\mathrm{u}$ and d quarks, it is subject to the periodic boundary condition. Recall that, due to the finite size of the lattice,

\begin{tabular}{lccc}
\hline \hline & $\mathrm{u}$ quark & $\mathrm{d}$ quark & $\mathrm{s}$ quark \\
\hline HBC & anti-periodic & anti-periodic & periodic \\
standard BC & periodic & periodic & periodic \\
\hline \hline
\end{tabular}

TABLE V: The hybrid boundary condition (HBC) imposed on the quark fields. The second line shows the standard $\mathrm{BC}$ for comparison.

the allowed spatial momenta are quantized as

$$
p_{i}= \begin{cases}2 n_{i} \pi / L & \text { for periodic BC } \\ \left(2 n_{i}+1\right) \pi / L & \text { for anti-periodic BC }\end{cases}
$$

with $n_{i} \in \mathbb{Z}$. Hence, $\mathrm{N}$ and $\mathrm{K}$ acquire the non-zero minimum momenta as

$$
\left|\vec{p}_{\min }\right|=\frac{\sqrt{3} \pi}{L}
$$

even for the s-wave state. By comparing this with Eq. (14), we see that $\mathrm{HBC}$ can raise the NK threshold like the p-wave case. (See Eq. (14).) In Table III we also show NK thresholds for the HBC. Note that, unlike the standard BC, s-wave threshold and p-wave one coincide in the HBC. We see, in Table III that s-wave NK threshold is raised by about $200-250 \mathrm{MeV}$ in the range of $0.1210 \leq \kappa \leq 0.1240$ in our calculation.

On the other hand, a localized resonance $\Theta^{+}(u u d d \bar{s})$ can have zero momentum as $\left|\vec{p}_{\min }\right|=0$. (See Table VI) Therefore, the shift of $m_{\Theta}$ comes only through the change in its intrinsic structure. In this case, the shift is expected to be less significant than the shift due to the kinematic reason as is the case in $\mathrm{N}$ and $\mathrm{K}$. Now, if the spatial size of $\Theta^{+}$is sufficiently smaller than the spatial lattice size $L$, then it is safely expected that the mass of $\Theta^{+}$is insensitive to the change of the boundary condition.

\section{B. Numerical result on substance of $5 \mathrm{Q}$ state}

Before presenting the results for $\Theta^{+}$, we have to convince ourselves that localized resonance states are really insensitive to the change of boundary condition. For this purpose, we apply the HBC to the $\Sigma(u d s)$ baryon, which is an established localized resonance. We take $\Sigma$ rather than the nucleon, because $\Sigma$ contains even numbers of $u$ and $d$ quarks. Hence, $\Sigma$ is subject to the periodic boundary condition, and can have zero spatial momentum. Therefore, if the mass of $\Sigma$ is affected by the choice of the BC, it is attributed to a change of intrinsic structure. In Fig. 4 we show effective mass plots of $\Sigma$ baryon correlators adopting the typical set of hopping parameter Eq. (9). The upper one corresponds to the standard $\mathrm{BC}$, and the lower one to the HBC. We see that the plateau is raised by only a negligible amount due to the change of the boundary condition. This example explicitly shows that localized resonance states are insensitive to the change of the boundary condition. 


\begin{tabular}{lccll}
\hline \hline & quark content & spatial BC & minimum momentum & \\
\hline $\mathrm{N}$ & $u u d, u d d$ & anti-periodic & $\vec{p}_{\min }=\left( \pm \frac{\pi}{L}, \pm \frac{\pi}{L}, \pm \frac{\pi}{L}\right)$ & $\left|\vec{p}_{\min }\right|=\sqrt{3} \frac{\pi}{L}$ \\
$\mathrm{~K}$ & $u \bar{s}, d \bar{s}$ & anti-periodic & $\vec{p}_{\min }=\left( \pm \frac{\pi}{L}, \pm \frac{\pi}{L}, \pm \frac{\pi}{L}\right)$ & $\left|\vec{p}_{\min }\right|=\sqrt{3} \frac{\pi}{L}$ \\
$\Theta^{+}$ & $u u d d \bar{s}$ & periodic & $\vec{p}_{\min }=(0,0,0)$ & $\left|\vec{p}_{\text {min }}\right|=0$ \\
\hline \hline
\end{tabular}

TABLE VI: The consequence of the HBC on the hadrons.

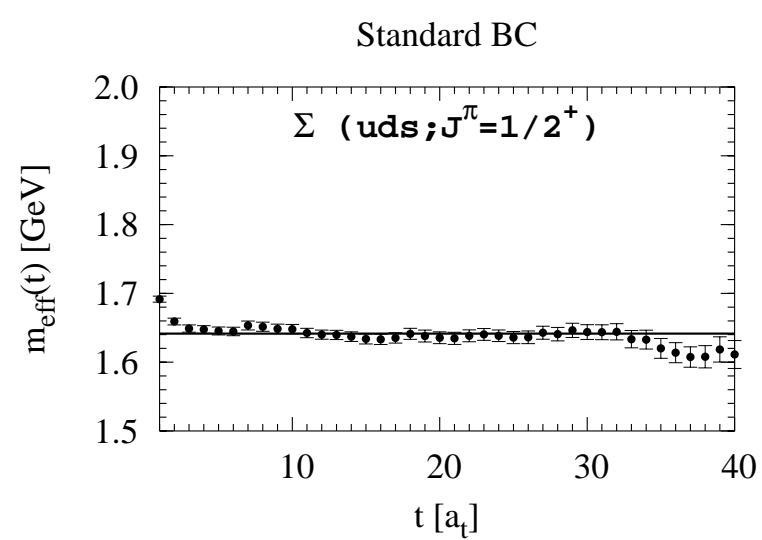

Hybrid BC

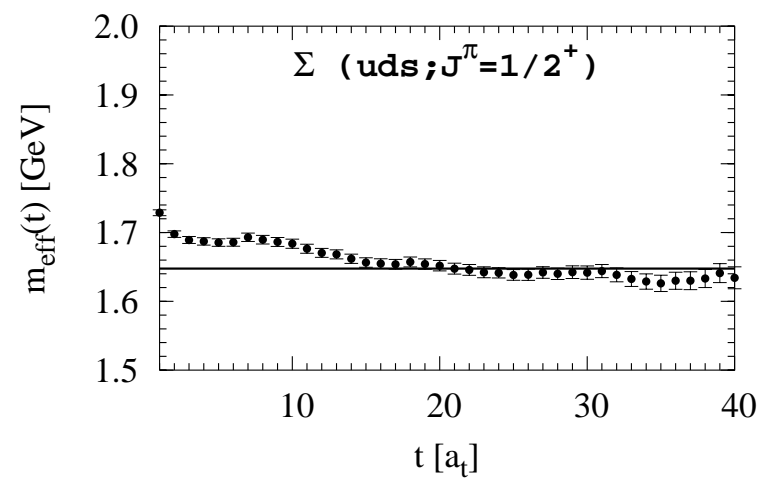

FIG. 4: The effective mass plots of $\Sigma(u d s)$ baryon under the standard BC, and under the HBC. Eq. (9) is adopted as a typical set of the hopping parameters. The solid lines denote the results of the single exponential fit performed in the region $20 \leq t \leq 30$. No significant difference is observed between the two results.

Now, we present the numerical result of negative parity $\Theta^{+}$. In Fig. 5 we show the effective mass of negative parity $\Theta^{+}$imposing the $\mathrm{HBC}$ adopting $\kappa_{s}=0.1240$ and $\kappa=0.1220$. In the region $0<t \lesssim 25$, the effective mass decrease monotonically as before. There is a plateau in the region $25 \lesssim t \lesssim 35$. Beyond $t \sim 35$, the plateau breaks down due to the contributions from backwardly-propagating positive parity states. Hence, we simply neglect the data for $t \lesssim 35$, and perform the single-exponential fit in the plateau region, $25 \leq t \leq 35$. The solid line denotes the result of the single-exponential fit. The dotted line denotes the modified NK threshold, i.e., $\sqrt{m_{\mathrm{N}}^{2}+\vec{p}_{\min }^{2}}+\sqrt{m_{\mathrm{K}}^{2}+\vec{p}_{\min }^{2}}$ with $\left|\vec{p}_{\min }\right|=\sqrt{3} \pi / L$

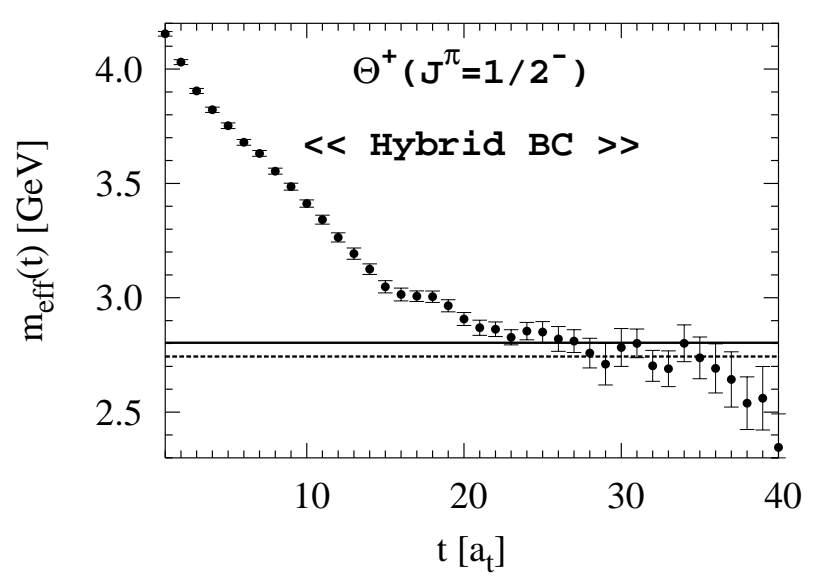

FIG. 5: The effective mass plot for the negative parity $\Theta^{+}$ under the HBC. Eq. (9) is adopted as a typical set of hopping parameters. The solid line denotes the result of the singleexponential fit performed within the interval $25 \leq t \leq 35$. The dotted line denotes the s-wave NK threshold energy modified due to the HBC. This figure should be compared with Fig. 2

due to the HBC. Comparing Fig. 5 with Fig. 2 we observe that the plateau is raised by about $200 \mathrm{MeV}$, which coincides with the shift of the NK threshold. In Fig. 6] we compare the result of $\mathrm{HBC}$ with that of standard $\mathrm{BC}$ for each hopping parameter $\kappa$ for the negative parity $\Theta^{+}$. Closed circles denote the results obtained with best-fit analysis. Solid lines show the corresponding NK thresholds. For all $\kappa$, we see that the best-fit masses $(\simeq$ the location of the plateaus) are raised by about the same amount as the corresponding NK thresholds. Therefore, we find that there is no localized resonance states below $\sqrt{m_{\mathrm{N}}^{2}+\vec{p}_{\text {min }}^{2}}+\sqrt{m_{\mathrm{K}}^{2}+\vec{p}_{\text {min }}^{2}}$ with $\left|\vec{p}_{\text {min }}\right|=\sqrt{3} \pi / L$. As a result, we conclude that the negative parity state observed before is an NK scattering state.

\section{Comments}

(i) The fully anti-periodic boundary condition is less convenient for our purpose. In fact, in this case, $\mathrm{K}$ is subject to the periodic boundary condition, whereas $\mathrm{N}$ and $\Theta^{+}$are subject to the anti-periodic boundary condition. Since $\mathrm{K}$ can have the zero momentum, the shift of the NK threshold is smaller. In addition, $\Theta^{+}$must have 


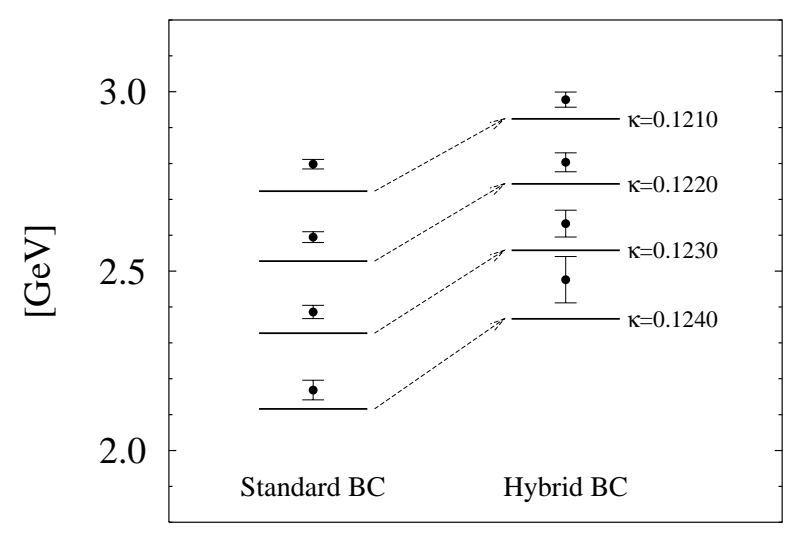

FIG. 6: Comparison of results of standard BC(l.h.s.) and HBC(r.h.s.) for each hopping parameter $\kappa$. Closed circles denote results obtained from the best fit analysis. The solid lines denote the corresponding NK threshold.

non-zero momentum, which raises the location of $\Theta^{+}$due to the kinematic reason. It is easy to understand that the resulting spectrum is less convenient for our purpose.

(ii) It is possible to use the HBC in the study of the positive parity state. However, since the change of the minimum momenta amounts to only a minor modification from $2 \pi / L$ to $\sqrt{3} \pi / L$, the resulting change in the spectrum is less significant than the negative parity state.

(iii) The HBC provides us with a general tool, the use of which is not restricted to the calculation of $\Theta^{+}$. For instance, it can be used in the study of the hadronic molecules. Hadronic molecules are bound state of two or more hadrons. The binding energies are typically $10-50$ $\mathrm{MeV}$. In the lattice QCD studies, the gap of $10-50$ $\mathrm{MeV}$ may be too small to confirm the existence of the bound state. Therefore the $\mathrm{HBC}$ is useful by enlarging this gap to a few hundred $\mathrm{MeV}$.

(iv) Careful reader may point out that, besides the stable plateau which we discussed, there is a small plateaulike structure in Fig. 2 and Fig. 5 in the region $15 \leq$ $t \leq 18$. However, this "plateau" is also raised by about $200 \mathrm{MeV}$ due to the HBC. This means that this region is not dominated by a single state, and a possible reason of this shift would be that contaminations of low-lying states becomes reduced in the HBC. Still, the nature of this small plateau cannot be determined from our data available so far. At any rate, we can state that it does not correspond to the experimentally observed $\Theta^{+}(1540)$, because its location is rather high $m \simeq 1.95 \mathrm{GeV}$ even after the chiral extrapolation. Note that we do not find such a plateau-like structure in the effective mass plot with a point source and a point sink.

\section{SUMMARY AND DISCUSSION}

We have studied the penta-quark baryon $\Theta^{+}$in anisotropic lattice QCD at the quenched level. We have used the standard plaquette action for gauge field configurations on the anisotropic lattice of the size $12^{3} \times 96$ with the renormalized anisotropy $a_{s} / a_{t}=4$ at $\beta=$ 5.75 , i.e., $a_{s} \simeq 0.18 \mathrm{fm}, a_{t} \simeq 0.045 \mathrm{fm}$. For quarks, we have adopted $O(a)$-improved Wilson (clover) action with four values of hopping parameters as $\kappa=$ $0.1210(0.0010) 0.1240$. To enhance the low-lying spectra, we have adopted a spatially extended operator.

We have used 504 gauge field configurations to construct the correlator of a non-NK type interpolating field. For each parity channel, we have found a rather stable plateau, where the single exponential fit has been performed. After the chiral extrapolation, we have found $m_{\Theta}=2.25 \mathrm{GeV}$ for positive parity state, which is too heavy to be accepted as the experimentally observed $\Theta^{+}(1540)$. For negative parity case, we have obtained $m_{\Theta}=1.75 \mathrm{GeV}$, which is rather close to the empirical value. Our data have thus suggested that the negative parity state is the lowest of the penta-quark spectrum.

In order to clarify whether this negative parity state is a localized resonance or not, we have proposed a new method with "hybrid boundary condition (HBC)", where the anti-periodic spatial boundary condition is imposed on the $\mathrm{u}$ and $\mathrm{d}$ quarks, while the periodic spatial boundary condition is imposed on the s quark. As results, $N(u u d, u d d)$ and $K(u \bar{s}, d \bar{s})$, both of which contain odd numbers of $\mathrm{u}$ and $\mathrm{d}$ quarks, are subject to the anti-periodic spatial boundary condition, whereas $\Theta^{+}(u u d d \bar{s})$, which contains even numbers of the $\mathrm{u}$ and $\mathrm{d}$ quarks, is subject to the periodic boundary condition. A remarkable feature of the $\mathrm{HBC}$ is that it raises the s-wave NK threshold by a few hundred $\mathrm{MeV}$ without affecting the localized penta-quark resonance.

By using the HBC method, we have further investigated the negative parity state. We have found that the plateau is raised by about $200 \mathrm{MeV}$ due to the HBC, which is consistent with the shift of the NK threshold. We conclude that there is no localized resonance state below $\sqrt{m_{\mathrm{N}}^{2}+\vec{p}_{\text {min }}^{2}}+\sqrt{m_{\mathrm{K}}^{2}+\vec{p}_{\text {min }}^{2}}$ with $\left|\vec{p}_{\text {min }}\right|=\sqrt{3} \pi / L$. It follows, in particular, that the negative parity state observed in the standard $\mathrm{BC}$ is a mere NK scattering state.

Now we discuss possible implications of our results. Experimentally, a number of groups [1, 6, 7, 8, 9] have confirmed the existence of $\Theta^{+}(1540)$. Hence, it should be observed in lattice QCD Monte Carlo calculation as well. However, in neither positive nor negative parity states, we have found any relevant signals of narrow penta-quark states. In this way, we have arrived at a similar result as Ref. 45]. However, it should be remarked that this result has been obtained in spite of our choice of the non-NK type interpolating field. These two results suggest that $\Theta^{+}$may not be reachable by a simple five-quark interpolating field operator in lattice QCD —it might have 
more complicated structure than expected. Needless to say, it is desirable to examine the finite volume artifact and discretization error, as usual. It is also desirable to reduce the ambiguity from the chiral extrapolation. Concerning the light quark effects, one might wonder that the dynamical quark effects might be important, because the narrow resonance $\Theta^{+}$was originally predicted by the Skyrme model [2]. After all, the Skyrmion is a nontrivial configuration of pion. However, this argument is not quite correct, since the nucleon serves as a counterexample. A nucleon is indeed the ground state of in the Skyrme model and thus contains a non-trivial configuration of pion. It is however also fairly well reproduced in quenched lattice QCD with conventional three-quark interpolating field.

Besides these rather technical stuff, the difficulty may trace back to our insufficient understanding of the true nature of the penta-quark. It would be interesting to consider the following possibilities.

(1) Highly non-local structure: If the structure of $\Theta^{+}$is really the Jaffe-Wilczek type or the Karliner-Lipkin type, it may be better to invent series of new non-local interpolating fields, which have sufficiently complicated structures so that they can fit Jaffe-Wilczek/Kaliner-Lipkin pictures. In this respect, the group theoretical construction of interpolating fields proposed in Ref. 53. together with the variational method would be interesting.

(2) Other quantum numbers: In most lattice QCD calculations of $\Theta^{+}$, spin of $\Theta^{+}$is assumed to be $1 / 2$. Naively, one may expect that $J=3 / 2$ state would be heavier than $\mathrm{J}=1 / 2$ states. However, since there is no experimental evidence on the spin of $\Theta^{+}$, it would be worth keeping $J=3 / 2$ and $5 / 2$ possibilities as candidates. We have to keep in mind that $\Theta^{+}$is already a quite mysterious particle. In addition, although there is no evidence on the doubly charged $\Theta^{++}$in the experiment $[8]$, it might be worth studying $\mathrm{I}=1$ and 2 states as well, which have been suggested by Ref. 36]. Since we do not know the production mechanism of the penta-quark, it might be better to keep them as possible candidates.

(3) $K \pi N$ hepta-quark hypothesis: All the lattice QCD calculations for $\Theta^{+}$available so far are based on the quenched QCD. It is known that some hadrons are difficult to be reproduced in the quenched QCD. For instance, quenched lattice QCD cannot reproduce $\Lambda(1405)$ as a three-quark state [50]. It is conjectured to be a $\bar{K} N$ bound state. In this case, $\Lambda(1405)$ is a "penta-quark". Hence, it would be natural that mass of $\Lambda(1405)$ cannot be properly reproduced in quenched lattice QCD with a standard three-quark interpolating field. Actually, $\Theta^{+}(1540)$ itself is hypothesized to be a bound state of $K \pi N$, i.e., the hepta-quark 21, 22, 54]. If this is really the case, it would be natural that $\Theta^{+}(1540)$ is difficult to be observed in quenched lattice QCD with "ordinary" penta-quark interpolating fields. However, concerning the hepta-quark picture, we should keep in mind that, in spite of its many good features, the interaction among $K \pi N$ system is said to be too weak to make a bound state with the binding energy of $40 \mathrm{MeV}$.

In this way, it is necessary to perform more systematic studies of the penta-quarks in order to reveal its mysterious nature. The proper use of lattice QCD can help us proceed in this direction.

\section{Acknowledgments}

We thank H. Matsufuru, Y. Nemoto, T.T. Takahashi and T. Umeda for useful information and discussions. M. O and H. S are supported in part by Grant for Scientific Research ((B) No. 15340072 and (C) No. 16540236) from the Ministry of Education, Culture, Sports, Science and Technology, Japan. T. D. is supported by Special Postdoctoral Research Program of RIKEN. H. I. is supported by a 21st Century COE Program at Tokyo Institute of Technology "Nanometer-Scale Quantum Physics" by the Ministry of Education, Culture, Sports, Science and Technology. The lattice QCD Monte Carlo calculations have been performed on NEC-SX5 at Osaka University.

\section{APPENDIX A: REARRANGEMENTS OF EQ. (1)}

The aim here is to estimate the contribution from NKtype operator to the correlator of Eq. (11). To this end, we use the Fierz rearrangements. By using an identity $\epsilon_{a b c} \epsilon_{b f g}=\delta_{c f} \delta_{a g}-\delta_{c g} \delta_{a f}$, and by applying the Fierz identity to $\left(u_{f}^{T} C d^{g}\right) C \bar{s}_{c}^{T}$, Eq. (1) is rearranged as

$$
\begin{aligned}
\epsilon_{a b c} \epsilon_{a d e} \epsilon_{b f g}\left(u_{d}^{T} C \gamma_{5} d_{e}\right)\left(u_{f}^{T} C d_{g}\right) C \bar{s}_{c}^{T} \\
=\epsilon_{a d e}\left(u_{d}^{T} C \gamma_{5} d_{e}\right)\left\{\left(u_{c}^{T} C d_{a}\right) C \bar{s}_{c}^{T}-\left(u_{a}^{T} C d_{c}\right) C \bar{s}_{c}^{T}\right\} \\
=\epsilon_{a d e}\left(u_{d}^{T} C \gamma_{5} d_{e}\right) \\
\quad \times \frac{1}{4}\left\{-\gamma_{5} d_{a}\left(\bar{s}_{c} \gamma_{5} u_{c}\right)+\gamma_{5} u_{a}\left(\bar{s}_{c} \gamma_{5} d_{c}\right)\right. \\
\quad-d_{a}\left(\bar{s}_{c} u_{c}\right)+u_{c}\left(\bar{s}_{c} d_{c}\right) \\
\quad+\gamma_{\mu} d_{a}\left(\bar{s}_{c} \gamma_{\mu} u_{c}\right)-\gamma_{\mu} u_{a}\left(\bar{s}_{c} \gamma_{\mu} d_{c}\right) \\
\quad+\gamma_{5} \gamma_{\mu} d_{a}\left(\bar{s}_{c} \gamma_{5} \gamma_{\mu} u_{c}\right)-\gamma_{5} \gamma_{\mu} u_{a}\left(\bar{s}_{c} \gamma_{5} \gamma_{\mu} d_{c}\right) \\
\left.\quad+\frac{1}{2} \sigma_{\mu \nu} d_{a}\left(\bar{s}_{c} \sigma_{\mu \nu} u_{c}\right)-\frac{1}{2} \sigma_{\mu \nu} u_{a}\left(\bar{s}_{c} \sigma_{\mu \nu} d_{c}\right)\right\}
\end{aligned}
$$

where only the first and the second term factorize into the product of the interpolating fields of $\mathrm{N}$ and $\mathrm{K}$ up to a factor of $\gamma_{5}$. We see that Eq. (1) contains the NK-type interpolating field with a factor of $\frac{1}{4}$.

In Eq. A1, $\mathrm{u}$ and d fields in K come from the pseudoscalar diquark operator. There is another contribution, where $\mathrm{u}$ and $\mathrm{d}$ fields in $\mathrm{K}$ come from the scalar diquark operator. This is obtained by using the identity $\epsilon_{a b c} \epsilon_{a d e}=\delta_{b d} \delta_{c e}-\delta_{b e} \delta_{c d}$ and by applying the Fierz iden- 
tity to $\left(u_{d}^{T} C \gamma_{5} d_{3}\right) C \bar{s}_{c}^{T}$ in the following way:

$$
\begin{aligned}
\epsilon_{a b c} \epsilon_{a d e} \epsilon_{b f g}\left(u_{d}^{T} C \gamma_{5} d_{e}\right)\left(u_{f}^{T} C d_{g}\right) C \bar{s}_{c}^{T} \\
=\epsilon_{b f g}\left(u_{f}^{T} C d_{g}\right)\left\{\left(u_{b}^{T} C \gamma_{5} d_{c}\right) C \bar{s}_{c}^{T}-\left(u_{c}^{T} C \gamma_{5} d_{b}\right) C \bar{s}_{c}^{T}\right\} \\
=\epsilon_{b f g}\left(u_{f}^{T} C d_{g}\right) \\
\quad \times \frac{1}{4}\left\{-u_{b}\left(\bar{s}_{c} \gamma_{5} d_{c}\right)+d_{b}\left(\bar{s}_{c} \gamma_{5} u_{c}\right)\right. \\
\quad-\gamma_{5} u_{b}\left(\bar{s}_{c} d_{c}\right)+\gamma_{5} d_{b}\left(\bar{s}_{c} u_{c}\right) \\
\quad-\gamma_{5} \gamma_{\mu} u_{b}\left(\bar{s}_{c} \gamma_{\mu} d_{c}\right)+\gamma_{5} \gamma_{\mu} d_{b}\left(\bar{s}_{c} \gamma_{\mu} u_{c}\right) \\
\quad-\gamma_{\mu} u_{b}\left(\bar{s}_{c} \gamma_{5} \gamma_{\mu} d_{c}\right)+\gamma_{\mu} d_{b}\left(\bar{s}_{c} \gamma_{5} \gamma_{\mu} u_{c}\right) \\
\left.\quad+\frac{1}{2} \sigma_{\mu \nu} u_{b}\left(\bar{s}_{c} \sigma_{\mu \nu} d_{c}\right)-\frac{1}{2} \sigma_{\mu \nu} d_{b}\left(\bar{s}_{c} \sigma_{\mu \nu} u_{c}\right)\right\},
\end{aligned}
$$

[1] LEPS Collaboration, T. Nakano et al., Phys. Rev. Lett. 91, 012002 (2003).

[2] D. Diakonov, V. Petrov and M.V. Polyakov, Z. Phys. A359, 305 (1997)

[3] R.L. Jaffe, SLAC-PUB-1774 (1976).

[4] D. Strottman, Phys. Rev. D20, 748 (1979).

[5] M. Praszalowicz, Phys. Lett. B575, 234 (2003); M. Praszalowicz, in : M. Jezabek, M. Praszalowicz (Eds.), Proceedings of the Workshop on Skyrmions and Anomalies, World Scientific, Singapole, 1987, p. 112.

[6] DIANA Collaboration, V.V. Barmin et al., Phys. Atom. Nucl. 66, 1715 (2003).

[7] CLAS collaboration, S. Stepanyan et al., Phys. Rev. Lett. 91, 252001 (2003).

[8] SAPHIR Collaboration, J. Barth et al., Phys. Lett. B572, 127 (2003).

[9] A.E. Asratyan, A.G. Dolgolenko, M.A. Kubantsev, Phys. Atom. Nucl. 67, 682 (2004); CLAS Collaboration, V. Kubarovsky, et al., Phys. Rev. Lett. 92, 032001 (2004); HERMES Collaboration, A. Airapetian, et al., Phys. Lett. B585, 213 (2004); SVD Collaboration, A. Aleev, et al., hep-ex/0401024 COSY-TOF Collaboration, M. Abdel-Bary, et al., Phys. Lett. B595, 127 (2004); ZEUS Collaboratioin, S. Chekanov, et al., Phys. Lett. B591, 7 (2004).

[10] T.D. Cohen, Phys. Lett. B581, 175 (2004).

[11] N. Itzhaki, I.R. Klebanov, P. Ouyang, L. Rastelli, Nucl. Phys. B684, 264 (2004).

[12] H.-Ch. Kim, Phys. Lett. B585, 99 (2004).

[13] A. Hosaka, Phys. Lett. B571, 55 (2003).

[14] R.L. Jaffe and F. Wilczek, Phys. Rev. Lett. 91, 232003 (2003).

[15] M. Karliner and H.J. Lipkin, Phys. Lett. B575, 249 (2003).

[16] C.E. Carlson, C.D. Carone, H.J. Kwee, V. Nazaryan, Phys. Lett. B579, 52 (2004).

[17] Fl. Stancu and D.O. Riska, Phys. Lett. B575, 242 (2003).

[18] B.K. Jennings and K. Maltman, Phys. Rev. D69, 094020 (2004).

[19] L.Y. Glozman, Phys. Lett. B575, 18 (2003).

[20] Y. Kanada-Enyo, O. Morimatsu, and T. Nishikawa, hep-ph/0404144

[21] P. Bicudo, and G.M. Marques, Phys. Rev. D69, 011503 (2004).

[22] F.J. Llanes-Estrada, E. Oset, and V. Mateu, Phys. Rev. where only the first and the second terms contribute to the product of interpolating fields of $\mathrm{N}$ and $\mathrm{K}$ up to a factor of $\gamma_{5}$. The nucleon interpolating field here has an unfamiliar form. Another Fierz rearrangement of this nucleon interpolating field can make this into the familiar form, which provides an additional factor of $\frac{1}{4}$. Totally, the contribution of the NK-type interpolating field to Eq. (1) in this case involves the factor of $1 / 16$.

By neglecting the small contribution in Eq. (A2) for simplicity, the direct contribution of the NK-type interpolating field (in source and sink) to the correlator of Eq. (1) involves the factor of $1 / 16$, which is rather small.

C69, 055203 (2004).

[23] C.E. Carlson, C.D. Carone, H.J. Kwee and V. Nazaryan, Phys. Lett. B573, 101 (2003).

[24] F. Huang, Z.Y. Zhang, Y.W. Yu, and B.S. Zou, Phys. Lett. B586, 69 (2004).

[25] T. Shinozaki, S. Takeuchi and M. Oka, Talk given at International Workshop PENTAQUARK04, July 2004, SPring-8, Harima, Japan.

[26] S.-L. Zhu, Phys. Rev. Lett. 91, 232002 (2003).

[27] R.D. Matheus, F.S. Navarra, M. Nielsen, R. Rodrigues da Silva, and S.H. Lee, Phys. Lett. B578, 323 (2004).

[28] J. Sugiyama, T. Doi, and M. Oka, Phys. Lett. B 581, 167 (2004).

[29] Y. Oh, H. Kim, and S.H. Lee, Phys. Rev. D69, 094009 (2004).

[30] Y. Maezawa, T. Maruyama, N. Itagaki, and T. Hatsuda, hep-ph/0408056

[31] I.M. Narodetskii, Yu.A. Simonov, M.A. Trusov, and A.I. Veselov, Phys. Lett. B578, 318 (2004).

[32] M. Bando, T. Kugo, A. Sugamoto, and S. Terunuma, hep-ph/0405259

[33] H. Suganuma, T.T. Takahashi, F. Okiharu, and H. Ichie, Talk given at International Workshop PENTAQUARK04, July 2004, SPring-8, Harima, Japan.

[34] F. Okiharu, H. Suganuma, and T.T. Takahashi, hep-lat/0407001 H. Suganuma, T.T. Takahashi, F. Okiharu, and H. Ichie, Proc. of QCD Down Under, Adelaide, March 2004, Nucl. Phys. B(Proc. Suppl.) in press.

[35] C. Alexandrou, and G. Koutsou, hep-lat/0407005

[36] S. Capstick, P.R. Page and W. Roberts, Phys. Lett. B570, 185 (2003).

[37] T. Nakano, and K. Hicks, Mod. Phys. Lett. A19, 645 (2004).

[38] A.W. Thomas, K. Hicks and A. Hosaka, Prog. Theor. Phys. 111, 291 (2004).

[39] For recent review article, M. Oka, Prog. Theor. Phys. 112, 1 (2004), and references therein.

[40] S.L. Zhu, hep-ph/0407157, and references therein.

[41] H. Weigel, Eur. Phys. J. A2, 391, (1998).

[42] F. Csikor, Z. Fodor, S.D. Katz, and T.G. Kovacs, JHEP 0311, 070 (2003).

[43] S. Sasaki, hep-lat/0310014

[44] T.W. Chiu and T.H. Hsieh, hep-ph/0403020 and hep-ph/0404007 
[45] N. Mathur, F.X. Lee, A. Alexandru, C. Bennhold, Y. Chen, S.J. Dong, T. Draper, I. Horváth, K.F. Liu, S. Tamhankar, and J.B. Zang, hep-ph/0406196

[46] N. Ishii, T. Doi, H. Iida, M. Oka, F. Okihara and H. Suganuma, talk given at Lattice 2004; talk given at International Workshop PENTAQUARK04, July 2004, SPring8, Harima, Japan.

[47] T.T. Takahashi, T. Kunihiro, T. Onogi, and T. Umeda, Talk given at International Workshop PENTAQUARK04, July 2004, SPring-8, Harima, Japan, and private communication.

[48] T.R. Klassen, Nucl. Phys. B533, 557 (1998).
[49] H. Matsufuru, T. Onogi, and T. Umeda, Phys. Rev. D64, 114503 (2001).

[50] Y. Nemoto, N. Nakajima, H. Matsufuru, and H. Suganuma, Phys. Rev. D68, 094505 (2003).

[51] N. Ishii, H. Suganuma, and H. Matsufuru, Phys. Rev. D66, 094506 (2002); Phys. Rev. D66, 014507 (2002).

[52] I. Montvay and G. Münster, "Quantum Fields on a Lattice", (CambridgeUniversityPress, Cambridge, England, 1994), p. 1.

[53] LHP Collaboration, S. Basak et al., hep-lat/0312003

[54] T. Kishimoto, and T. Sato, hep-ex/0312003 